\title{
Visual Evoked Potentials in Operated Primary Congenital Glaucoma: A Cross-Sectional Comparative Study
}

\author{
Mai A Mohammed' \\ Mohamed Saad Morsy' \\ Nashwa Shehata Zeater (D) ${ }^{2}$ \\ Nader Bayoumi $\mathbb{I}^{\prime}$ \\ 'Ophthalmology Department, Faculty of \\ Medicine, Alexandria University, \\ Alexandria, Egypt; ${ }^{2}$ Resident of \\ Ophthalmology, Damanhour Eye \\ Hospital, Damanhour, Egypt
}

Background: To evaluate the VEP parameters in operated controlled primary congenital glaucoma (PCG) eyes and compare them to normal age similar children eyes.

Methods: A cross-sectional comparative study conducted on 34 (19 right) eyes of 26 (19 males) children operated for PCG and 30 (17 right) eyes of 22 (12 males) age similar control children in a university-based practice. All study participants were subjected to a standard protocol of examination and electrophysiological testing (single flash VEP response, light adapted, pupils not dilated), reporting on the P2 implicit time, N1-P1 and N2-P2 (amplitude). Results: The mean $\pm \mathrm{SD}$ of the age of the study children and controls was $43.22 \pm 33.2$ and $55.68 \pm 35.2$ months respectively $(\mathrm{p}=0.217)$. The mean \pm SD IOP and cup/disc ratio of the study children were $18.4 \pm 5.2$ and $5.2 \pm 3.3 \mathrm{mmHg}$ and $0.7 \pm 0.2$ and $0.3 \pm 0.3$ at presentation and at testing, respectively. The VEP testing was conducted after $21.5 \pm 21.3$ months of surgery for PCG. There was no statistically significant differences in P2, N1-P1 and N2-P2 between patients and controls ( $\mathrm{p}=0.941,0.916,0.945$, respectively). There was no statistically significant correlation between most of the clinical characteristics of the study eyes and any of the studied VEP parameters.

Conclusion: Operated controlled PCG eyes have VEP parameters that match their normal fellow children.

Keywords: primary congenital glaucoma, visual evoked potentials, optic nerve, electrophysiology

\section{Introduction}

Primary congenital glaucoma (PCG) is diagnosed, according to the childhood glaucoma research network (CGRN), by the presence of at least 2 criteria of elevated intraocular pressure (IOP above $21 \mathrm{mmHg}$ ), characteristic corneal pathology (corneal Oedema, enlarged corneal diameter, Haab's striae), optic nerve changes (increased cup/disc ratio, asymmetry of cup/disc ratio, neural Rim thinning) and visual field changes ${ }^{1}$. The reported incidence of PCG is variable, from 1 in 10,000 live births ${ }^{2}$ in some communities to 1 in 2500 live births in other communities. $^{3}$ PCG is a potentially blinding disease. Visual disability in PCG is multifactorial. Errors of refraction are an expected sequel to corneal enlargement and possible Haab's striae and scars resulting in irregular astigmatism ${ }^{4}$ as well as increased axial length with its antecedent myopic shift. ${ }^{4}$ The possible oedema and scarring of the cornea pose a media opacity that compounds visual loss. Finally, neuronal loss culminates the disease process and heralds the onset of irreversible
Correspondence: Mai A Mohammed

36 Kamaleldin Salah, Semouha,

Alexandria, Egypt

Tel +201221081590

Fax +2034244441

Email mai.abdalnabi@yahoo.com 
visual loss. Treatment when successful effects halting of neuronal damage with possible reversal of some optic nerve cupping. ${ }^{5}$ Assessment of the optic nerve appearance can be done both subjectively by clinical examination and objectively by imaging modalities such as the OCT. ${ }^{6,7}$ Assessment of optic nerve function can be done subjectively by clinical examination (eg, preferential looking, optokinetic nystagmus, etc) and objectively by electrophysiology, namely visual evoked potential (VEP) testing. ${ }^{8}$ Studies have shown that optic nerve damage in childhood glaucoma is associated with abnormal pattern reversal VEPs. ${ }^{9}$ The standard protocols of VEP used worldwide are the pattern-reversal VEPs, pattern onset/offset VEPs and flash VEPs. ${ }^{10}$ For uncooperative patients including children the most used are flash VEP. The flash VEP tracing demonstrates a number of deflections, namely negative $(\mathrm{N})$ waves and positive $(\mathrm{P})$ waves, the most useful practically of which are the P2 implicit time, N1P1 and N2-P2 (amplitude). ${ }^{11}$ The current study was conducted to evaluate the flash VEP parameters in operated controlled PCG eyes and compare them to normal age similar children eyes.

\section{Methods}

The study was conducted on 34 (19 right) eyes of 26 (19 males) children with PCG that were operated upon in the ophthalmology department of Alexandria Main University Hospital and attending for regular follow up and 30 (17 right) eyes of 22 (12 males) age similar control children attending the pediatric ophthalmology practice of the ophthalmology Department of Alexandria Main University Hospital for a routine ophthalmic check. The study adhered to the tenets of the declaration of Helsinki and Institutional Review Board (the ethical committee of Alexandria University Faculty of Medicine) approval was obtained. Informed written consent was obtained from the parents/care providers of the study participants. All eyes operated for PCG in which the IOP was controlled were eligible for inclusion in the study. Poor control of the PCG and secondary childhood glaucoma were exclusion criteria (inclusion criteria included children diagnosed with PCG, and exclusion criteria included all secondary childhood glaucoma according to the CGRN definitions). Control children were recruited from age similar children that presented for a routine ophthalmic check for a number of complaints (eg, refractive error, ocular deviation, screening by the attending ophthalmologist). All study participants were subjected to a standard protocol of office examination, followed by examination under anaesthesia (EUA) (previously reported by the authors ${ }^{12}$ and included IOP measurement by Perkins tonometer, corneal measurement of horizontal corneal diameter, central corneal thickness [Pachmate, DGH technology], fundus examination including optic nerve assessment by indirect ophthalmoscopy and sonographic measurement of ocular axial length) and scheduled (the PCG cases) within one week for electrophysiological testing (flash VEP response, the pattern VEP was not used in this study to avert potential fixation problems and lack of sufficient cooperation of the study children). Flash VEP was conducted using the Roland Consult electrophysiology and imaging. The test was conducted with the child resting comfortably in the arms of the mother/familiar care provider and with the untested eye patched (the patch used was a black plastic eye shield that was carefully applied to the untested eye to ensure complete isolation from the test flash). If the child was cooperative enough an attempt was made to test both eyes (to use the patients' normal fellow eye as the patient's internal control) whenever this was possible. However, only the eye with operated controlled PCG was included for data analysis and statistical workup. The electrodes were placed according to the International society of Electrophysiology of vision System (ISCEV) standards ${ }^{10}$ and the pupils were not dilated (to simulate real-life situation as close as possible). The white flash stimulus was delivered using a mini ganzfeld stimulator to be easily handled with children. The VEPs were measured and recorded by an evoked potential inspection device, and were processed by averaging (about 100 stimuli) because potentials were extremely small. Peaks were designated as negative and positive in a numerical sequence (N1, P1, N2, P2, N3 and P3). Statistical workup was conducted using Microsoft EXCEL. Qualitative data were described using number and percent. Quantitative data were described using range (minimum and maximum), mean, standard deviation and median. Pearson correlation coefficient was used to study correlations while student $t$-test was used to compare groups. Significance of the obtained results was judged at the 5\% level. Correlations were sought between VEP parameters and the examination parameters, both at presentation (preoperative) and at the time of VEP testing (postoperatively).

\section{Results}

The study was conducted on 34 (19 right) eyes of 26 (19 males) children with PCG that were operated upon in the 
ophthalmology department of Alexandria Main University Hospital and attending for regular follow up and 30 (17 right) eyes of 22 (12 males) age similar control children. Demographic characteristics of the study patients and control children are presented in Table 1 . The mean age of the control children was slightly higher than the study children, though not statistically significant $(p=0.217)$. One fourth of the study children were born to consanguineous parents. One third of the study children were born with normal vaginal delivery (the remaining children born with Caesarian section); none reported any birth-related events (no perinatal hypoxia) and none of the study children was admitted to the neonatal intensive care unit (NICU). The clinical characteristics of the study eyes are presented in Table 2. At the time of the recording the mean \pm standard deviation IOP of the study eyes was $5.2 \pm 3.3 \mathrm{mmHg}$ and the cup/disc ratio was $0.3 \pm 0.3$ demonstrating significant improvement than at presentation. Two study eyes demonstrated a relative afferent papillary defect on clinical examination. The fellow eyes of the study children demonstrated absence of PCG at the time of the study as evidenced by a mean \pm standard deviation of the IOP, cup/ disc ratio and axial length of $8.8 \pm 4.7 \mathrm{mmHg}, 0.2 \pm 0.2$ and $23.09 \pm 1.22 \mathrm{~mm}$, respectively. The recordings were conducted around 2 years after the surgical intervention for PCG. The VEP findings of the study and control eyes are presented in Table 3 . There was no statistically significant differences in P2, N1-P1 and N2-P2 in the study eyes ( $p=0.941,0.916,0.945$, respectively) between patients and controls. The correlations between the study eye clinical characteristics at presentation (preoperative) and at recording are presented in Table 4 and Figure 1 presents

Table I Study Patients and Controls Demographic Characteristics

\begin{tabular}{|c|c|c|}
\hline \multicolumn{3}{|l|}{ Patients/Controls Demographic Characteristics } \\
\hline & Patients & Controls \\
\hline Study Participants (n,\%) & $26(100 \%)$ & $22(100 \%)$ \\
\hline Male & $19(73 \%)$ & $12(55 \%)$ \\
\hline Female & $7(27 \%)$ & $10(45 \%)$ \\
\hline Laterality of Study Eyes (n,\%) & $34(100 \%)$ & $30(100 \%)$ \\
\hline Right & $19(56 \%)$ & $17(57 \%)$ \\
\hline Left & $15(44 \%)$ & $13(43 \%)$ \\
\hline Age at Test (mean $\pm S D$, range) (months) & $43.22 \pm 33.2,9.8-145.8$ & $55.68 \pm 35.2,6.4-128.6$ \\
\hline Age at Presentation (patients) (mean $\pm S D$, range) (months) & $10.73 \pm 12.4,2.0-60.0$ & \\
\hline Patients born to consanguineous parents $(n, \%)$ & $7(27 \%)$ & \\
\hline Patients born with normal vaginal delivery/CS (n,\%) & $8 / 18(31 \% / 69 \%)$ & \\
\hline
\end{tabular}

Abbreviation: CS, caesarian section.

Table 2 Study Eyes Clinical Characteristics

\begin{tabular}{|c|c|c|}
\hline \multicolumn{3}{|l|}{ Study Eyes Clinical Characteristics } \\
\hline & At Presentation & At VEP Test \\
\hline IOP (mean $\pm S D$, range) $(\mathrm{mmHg})$ & $|8.4 \pm 5.2,8-3|$ & $5.2 \pm 3.3,0-13$ \\
\hline Corneal Diameter (mean $\pm S D$, range) $(\mathrm{mm})$ & $13.9 \pm 0.7,13-15.5$ & $14.2 \pm 0.7,13-16$ \\
\hline Axial Length (mean $\pm S D$, range) $(\mathrm{mm})$ & $24.66 \pm 2.45,21.12-32.87$ & $24.55 \pm 2.65,19.82-32.79$ \\
\hline Cup/Disc ratio(mean $\pm S D$, range) & $0.7 \pm 0.2,0.3-1.0$ & $0.3 \pm 0.3,0.0-1.0$ \\
\hline Duration from Surgery to Scan (mean $\pm S D$, range) (months) & \multicolumn{2}{|c|}{$21.5 \pm 21.3(1.0-60.0)$} \\
\hline Right eyes & \multicolumn{2}{|c|}{$38.1 \pm 31.6(1.2-113.9)$} \\
\hline Left eyes & \multicolumn{2}{|c|}{$26.5 \pm 30.8(1.6-116.3)$} \\
\hline
\end{tabular}


Table 3 Study Patients (Affected Eyes) and Controls Visual Evoked Potentials

\begin{tabular}{|l|c|c|c|}
\hline \multirow{2}{*}{ Total affected eyes (right eyes and left eyes data combined) } & \multicolumn{3}{|c|}{ Patients/Controls Visual Evoked Potentials } \\
\cline { 2 - 4 } & (Mean \pm SD, Range) & Patients & Controls \\
\hline & P2 & $117.10 \pm 24.4,75.1-162.5$ & $110.46 \pm 9.1,90.2-124.0$ \\
& N2-P2 & $8.31 \pm 9.8,0.38-53.4$ & $5.0 \pm 7.5,0.3-40.2$ \\
& $17.90 \pm 12.5,1.8-67.7$ & $24.32 \pm 15.2,5.7-78.6$ \\
\hline
\end{tabular}

Table 4 Study Eyes Clinical Characteristics Correlations with VEP Parameters

\begin{tabular}{|c|c|c|c|c|c|c|c|c|c|c|c|c|}
\hline \multicolumn{13}{|c|}{ Study Eyes Clinical Characteristics Correlations with VEP Parameters } \\
\hline & \multicolumn{6}{|c|}{ At Presentation } & \multicolumn{6}{|c|}{ At VEP Test } \\
\hline & \multicolumn{2}{|c|}{$\mathbf{P 2}$} & \multicolumn{2}{|c|}{ NI-PI } & \multicolumn{2}{|c|}{ N2-P2 } & \multicolumn{2}{|c|}{$\mathbf{P 2}$} & \multicolumn{2}{|c|}{ NI-PI } & \multicolumn{2}{|c|}{ N2-P2 } \\
\hline & $\boldsymbol{r}$ & $p$ & $\boldsymbol{r}$ & $p$ & $\boldsymbol{r}$ & $p$ & $\boldsymbol{r}$ & $p$ & $\boldsymbol{r}$ & $\mathbf{p}$ & $\boldsymbol{r}$ & $p$ \\
\hline IOP $(\mathrm{mmHg})$ & & & & & & & & & & & & \\
\hline OD & -0.15 & 0.507 & -0.21 & 0.353 & -0.23 & 0.313 & 0.07 & 0.743 & 0.05 & 0.823 & 0.04 & 0.862 \\
\hline OS & 0.35 & 0.137 & 0.05 & 0.796 & -0.003 & 0.987 & 0.104 & 0.670 & 0.07 & 0.753 & -0.32 & 0.158 \\
\hline Total affected & -0.17 & $0.46 \mathrm{I}$ & -0.07 & 0.760 & -0.14 & 0.535 & 0.15 & 0.512 & 0.17 & $0.44 I$ & 0.32 & 0.159 \\
\hline \multicolumn{13}{|l|}{ Corneal Diameter $(\mathrm{mm})$} \\
\hline OD & -0.17 & 0.462 & 0.05 & 0.809 & -0.14 & 0.518 & -0.33 & 0.134 & -0.30 & 0.184 & -0.46 & $0.035^{*}$ \\
\hline OS & -0.12 & 0.625 & -0.02 & 0.924 & 0.06 & 0.765 & -0.18 & 0.444 & 0.04 & 0.860 & -0.24 & 0.285 \\
\hline Total affected & 0.12 & 0.582 & 0.30 & 0.178 & -0.03 & 0.877 & 0.17 & 0.436 & 0.22 & 0.327 & 0.22 & 0.323 \\
\hline \multicolumn{13}{|l|}{ Axial Length (mm) } \\
\hline OD & -0.23 & 0.311 & -0.03 & 0.878 & -0.07 & 0.769 & -0.19 & 0.395 & -0.07 & 0.735 & -0.08 & 0.713 \\
\hline OS & -0.17 & 0.462 & -0.18 & 0.426 & -0.18 & 0.416 & -0.31 & 0.188 & -0.05 & 0.819 & -0.32 & 0.156 \\
\hline Total affected & 0.15 & 0.514 & 0.06 & 0.774 & -0.13 & 0.547 & 0.28 & 0.217 & 0.21 & 0.358 & 0.08 & 0.730 \\
\hline \multicolumn{13}{|l|}{ Cup/Disc ratio } \\
\hline OD & 0.01 & 0.965 & 0.22 & 0.338 & -0.20 & 0.375 & -0.05 & 0.821 & 0.0002 & 0.999 & -0.06 & 0.765 \\
\hline OS & 0.10 & 0.683 & -0.16 & 0.499 & -0.13 & 0.566 & -0.17 & 0.468 & 0.09 & 0.682 & -0.27 & 0.229 \\
\hline Total affected & 0.002 & 0.990 & 0.15 & 0.502 & 0.15 & 0.506 & 0.23 & 0.299 & 0.02 & 0.898 & -0.07 & 0.755 \\
\hline $\begin{array}{l}\text { Duration from Surgery to Scan (mean } \\
\pm S D \text {, range) (months) }\end{array}$ & \multicolumn{12}{|c|}{$21.5 \pm 21.3(1.0-60.0)$} \\
\hline Right & \multicolumn{12}{|c|}{$38.1 \pm 31.6(1.2-113.9)$} \\
\hline Left & \multicolumn{12}{|c|}{$26.5 \pm 30.8(1.6-116.3)$} \\
\hline
\end{tabular}

the flash VEP recording studied parameters and the IOP and $\mathrm{C} / \mathrm{D}$ ratio. There was no statistically significant correlation between any of the clinical characteristics of the study eyes and any of the studied VEP parameters. A sample recording (case/control) is presented in Figure 2.

\section{Discussion}

The aim of this study was to report on the VEP findings in operated controlled PCG eyes and to compare this to age similar normal children. The studied VEP parameters were the P2, N1-P1 and N2-P2. The VEP findings in operated PCG eyes were not significantly different from the eyes of normal age-matched children. The numbers of study eyes and laterality distribution of cases and controls in this study were closely matched rendering the comparison of the results more valid and robust. There was a predominance of males in PCG cases (about 3 fourths of the study population) thus paralleling the predominant male prevalence of the disease already reported by the author $^{13}$ and others ${ }^{14}$ and contrary to other reports in which no gender predilection was found. ${ }^{15}$ The age of the study children was around 4 years for both PCG cases 


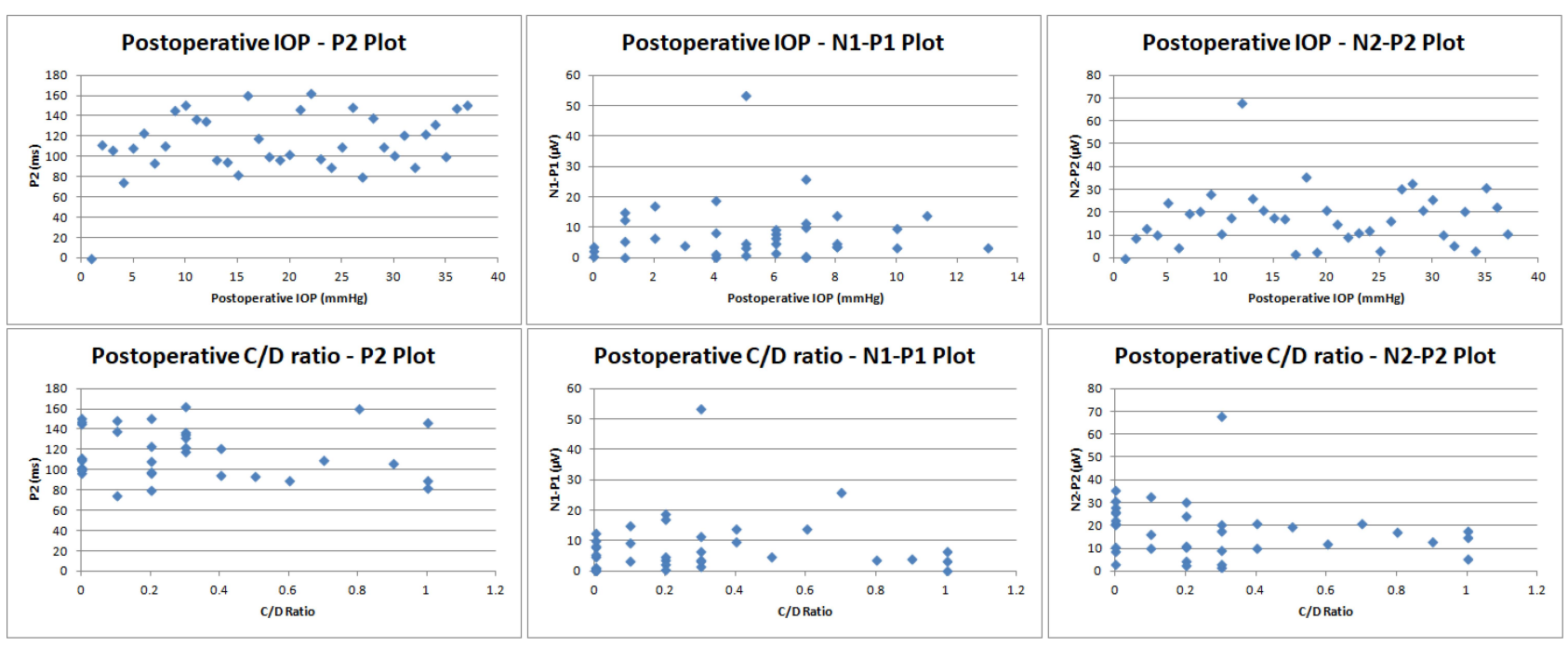

Figure I The flash VEP recording studied parameters and the IOP and C/D ratio.

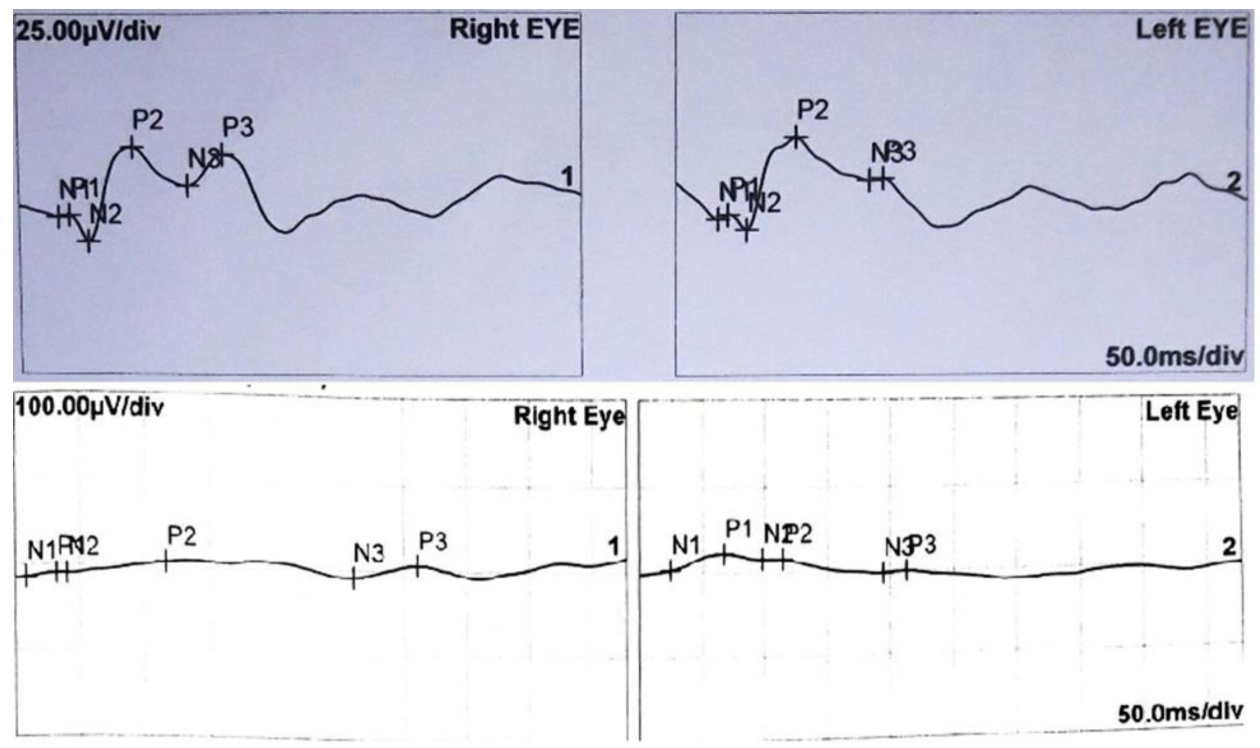

Figure 2 A sample recording (case/control).

and controls which allowed reasonable cooperation of the children during the VEP testing and obviated the need for the use of sedation during the testing. The average age of the study children and controls was similar to that reported for electrophysiological studies on children. ${ }^{16}$ The fact that general anaesthesia (GA) was not used during the VEP testing meant that even the pacified child in a mother's lap was needed to provide some cooperation during the testing. GA was not used during VEP testing to avoid any possible confounding effect on the VEP parameters by the CNS depression inherent to inhalational GA. ${ }^{17}$ Children with PCG in the current study presented at a slightly older age than the usual first 6 months of life common to $\mathrm{PCG},{ }^{14}$ although the clinical parameters at presentation of the study eyes do not reflect more severe disease than those in other reports. ${ }^{18}$ Strikingly, this relatively delayed presentation did not result in a deleterious effect on the optic nerve structure and function as evidenced by lack of statistically significant correlation between the age at presentation 
with any of the studied VEP parameters. This is in contrast to the report by Yadav et $\mathrm{al}^{19}$ who reported a significant reduction in $\mathrm{P} 2$ implicit time with advancing age in children. This difference may be attributed to the fact that the average age range of the current study was slightly older than the study by Yadav et al. The fact that only one fourth of the study children were born to consanguineous parents reflects the documented recessive inheritance nature and incomplete penetrance of PCG. ${ }^{20}$ An additional guarantee of absence of confounders for the VEP testing is the lack of any perinatal events or Neonatal Intensive Care Unit (NICU) admissions of all study children, ensuring that perinatal hypoxia was not present to potentially affect neurodevelopment and hence possibly the VEP results.

Scrutiny of the clinical data of the study eyes reveals that the PCG was controlled by surgery in all the study eyes at the time of the VEP testing, as evidenced by reduction of IOP and reversal of optic nerve cupping. The finding of an apparently low IOP value in study eyes is an already reported criterion of operated controlled $\mathrm{PCG}^{13}$ (and especially so when examined under GA with its antecedent effect on lowering IOP) and as reported in other studies has no deleterious effect on the visual functioning of the child, at least clinically. ${ }^{12}$ These clinical criteria of the study eyes are in accordance with other published reports. ${ }^{21}$ The mean duration between the VEP testing and the surgery for PCG was almost 2 years, a significant time to ensure reversal of any potentially reversible deleterious effect of PCG on optic nerve function. The reversibility of optic nerve cupping with successful treatment of PCG is reported by a number of studies. ${ }^{22,23}$ However, given the large variation in the duration between the VEP testing and PCG surgery, whether this duration has an effect on the VEP testing and whether the VEP testing outcome would be a function of this duration, remain speculative.

The ultimate standout finding of the current study is the VEP parameters of the study and control eyes. These VEP parameters demonstrate values in the current study that are close to those reported in other studies, ${ }^{19}$ thus precluding any effect of pupil diameter on the current study findings given that pupil dilation was not used. These parameters demonstrate no statistically significant differences between PCG patients' eyes and control eyes. These results suggest absence of a deleterious effect of PCG on optic nerve function following successful control of the disease by surgery, though this could never be confirmed without a longitudinal study of the same VEP parameters before and after surgery for PCG. To the best of the authors' knowledge, the comparison of the electrophysiological parameters of eyes with successful controlled PCG to age similar normal children eyes has not been reported. The observation that the $\mathrm{P} 2$ implicit time is slightly bigger in PCG patients' eyes than controls raises the possibility of mild delayed conduction along the ON of PCG eyes, a permanent stigma of PCG in afflicted eyes, even after recovery. This delayed conduction along the optic nerves of PCG eyes, despite control of the disease, parallels other reports $^{8}$ of VEP in glaucoma eyes. Other stigmata of PCG, albeit adequate control of the disease, are slightly exaggerated N1-P1 and N2-P2 amplitudes. Studying the correlations of the clinical parameters with the VEP parameters reveals a negative correlation between IOP and the VEP parameters, and between the C/D ratio and the VEP parameters, though both being statistically insignificant. The relatively small sample size of the study may be responsible for the correlations not reaching the level of statistical significance. This is in accordance with the study by Jha et $\mathrm{al}^{8}$ though reporting on adult glaucoma. Although the VEP testing yields multitudes of waveforms with different components, the authors choose to focus on the 3 most significant deflections of the VEP tracing, namely P2 implicit time, N1-P1 and N2-P2 amplitudes.

This study has limitations. The relatively small number of PCG eyes is a limitation. However, given the level of cooperation needed to conduct the testing, difficulty in recruitment of study children is expected. The VEP testing procedure was not tested and re-tested for reproducibility in every patient. Cooperation issues given the age group of the study participants obviously precluded such confirmatory procedure. Lack of an objective assessment of the optic nerve structure (by OCT for example) and hence an objective correlation to the VEP parameters is another issue. The focus of this study was the electrophysiological - as a clue to the function - rather than the anatomical characteristics of the operated PCG eyes, hence obviating the use of the OCT. Lack of refraction data for the study and control eyes and its correlation with the VEP parameters may have highlighted a possible effect of refractive errors on visual function. Visual function data (fixation pattern, nystagmus, etc) and its correlation with the flash VEP findings would add potential value to the study findings. Obviously, children cooperation and comprehension issues were the barriers for such information. 
To conclude, operated controlled PCG eyes have VEP parameters that match their normal fellow children thus providing an objective evidence of potentially intact optic nerve function in operated controlled PCG and absence of a deleterious effect of the disease on the optic nerve function.

\section{Research Involving Human Participants}

All procedures performed in studies involving human participants were in accordance with the ethical standards of Alexandria Faculty of Medicine ethics committee and with the 1964 Helsinki declaration and its later amendments. The ethical approval was obtained from the ethics committee of Alexandria Faculty of Medicine, Alexandria, Egypt. A written informed consent was obtained from all individual participants included in the study.

\section{Data Sharing Statement}

Available upon request from the corresponding author.

\section{Informed Consent}

Informed written consent was obtained from of the study participants.

\section{Consent to Publish}

Informed written consent was obtained from participants to publish the findings.

\section{Disclosure}

The authors report no conflicts of interest in this work.

\section{References}

1. Hoguet A, Grajewski A, Hodapp E, Chang TCP. A retrospective survey of childhood glaucoma prevalence according to Childhood Glaucoma Research Network classification. Indian J Ophthalmol. 2016;64(2):118-123. doi:10.4103/0301-4738.179716

2. Yu Chan JY, Choy BNK, Ng ALK, Shum JWH. Review on the management of primary congenital glaucoma. J Curr Glaucoma Pract. 2015;9:92-99. doi:10.5005/jp-journals-10008-1192

3. Al-Hazmi A, Awad A, Zwaan J, Al-Mesfer SA, Al-Jadaan I, AlMohammed A. Correlation between surgical success rate and severity of congenital glaucoma. $\mathrm{Br} \quad J$ Ophthalmol. 2005;89:449453. doi:10.1136/bjo.2004.047761

4. Morin JD, Bryars JH. Causes of loss of vision in congenital glaucoma. Arch Ophthalmol. 1980;98(9):1575-1576. doi:10.1001/ archopht. 1980.01020040427005

5. Badawi AH, Al-Muhaylib AA, Al Owaifeer AM, Al-Essa RS, AlShahwan SA. Primary congenital glaucoma: an updated review. Saudi J Ophthalmol. 2019;33:382-388. doi:10.1016/j.sjopt.2019.10.002
6. Srinivasan S, Addepalli UK, Rao HL, Garudadri CS, Mandal,AK. Spectral domain optical coherence tomography in children operated for primary congenital glaucoma Sangeetha Srinivasan1. $\mathrm{Br}$ J Ophthalmol. 2014;98(2):162-165. doi:10.1136/bjophthalmol-2012302486

7. El-Dairi M. Optical coherence tomography in the management of congenital glaucoma. $B r \quad J$ Ophthalmol. 2014;98:149-150. doi:10.1136/bjophthalmol-2013-303723

8. Jha MK, Thakur D, Limbu N, Badhu BP, Paudel BH. Visual evoked potentials in primary open angle glaucoma. $J$ Neurodegener Dis. 2017;2017:1-4. doi:10.1155/2017/9540609

9. Fil'chikova LI, Dubovskaia LA, Sidorenko EI, et al. [Visual evoked potentials in children with congenital glaucoma]. Vestn Oftalmol. 1992;108(1):15-18.

10. Odom JV, Bach M, Brigell M, et al. ISCEV standard for clinical visual evoked potentials: (2016 update). Doc Ophthalmol. 2016;133 (1):1-9. doi:10.1007/s10633-016-9553-y

11. Visual evoked potential [Internet]. [cited March 10, 2020]. Available from: https://www.aao.org/bcscsnippetdetail.aspx?id=45cef5ac-2f4e4b67-81ff-85f3fd02878c. Accessed May 15, 2021.

12. Bayoumi NHL. Deep sclerectomy in pediatric glaucoma filtering surgery. Eye. 2012;26(12):1548-1553. doi:10.1038/eye.2012.215

13. Bayoumi NL. Surgical management of primary congenital glaucoma in Egypt. J Egypt Ophthalmol Soc. 2016;109(2):85. doi:10.4103/ 2090-0686.193398

14. Tamçelik N, Atalay E, Bolukbasi S, Çapar O, Ozkok A. Demographic features of subjects with congenital glaucoma. Indian J Ophthalmol. 2014;62(5):565-569. doi:10.4103/0301-4738.126988

15. Papadopoulos M, Cable N, Rahi J, et al. The British infantile and childhood glaucoma (BIG) eye study. Investig Ophthalmol Vis Sci. 2007;48(9):4100-4106. doi:10.1167/iovs.06-1350

16. Bendschneider D, Tornow RP, Horn FK, et al. Retinal nerve fiber layer thickness in normals measured by spectral domain oct. $J \quad$ Glaucoma. 2010;19(7):475-482. doi:10.1097/IJG.0b013e3 $181 \mathrm{c} 4 \mathrm{~b} 0 \mathrm{c} 7$

17. Wu L, Zhao H, Weng H, Ma D. Lasting effects of general anesthetics on the brain in the young and elderly: "mixed picture" of neurotoxicity, neuroprotection and cognitive impairment. $J$ Anesth. 2019;33:321-335. doi:10.1007/s00540-019-02623-7

18. Mandal BPG, Gothwal VK, Reddy VM, et al. Safety and efficacy of simultaneous bilateral primary combined trabeculotomy-trabeculectomy for developmental glaucoma. Indian J Ophthalmol. 2002;50(1):13.

19. Yadav R, Poudel BH, Limbu N, Thakur D, Yadav SK. Normative data of visual evoked potential in children and correlation with age. Asian J Med Sci. 2015;7(2):39-43. doi:10.3126/ajms.v7i2.13135

20. Cascella R, Strafella C, Germani C, et al. The genetics and the genomics of primary congenital glaucoma. Biomed Res Int. 2015;2015:1-7. doi:10.1155/2015/321291

21. Senthil S, Badakere S, Ganesh J, et al. Profile of childhood glaucoma at a tertiary center in South India. Indian J Ophthalmol. 2019;67 (3):358-365. doi:10.4103/ijo.IJO_786_18

22. Wu SC, Huang SCM, Kuo CL, Lin KK, Lin SM. Reversal of optic disc cupping after trabeculotomy in primary congenital glaucoma. Can J Ophthalmol. 2002;37(6):337-341. doi:10.1016/S00084182(02)80003-5

23. Ely AL, El-Dairi MA, Freedman SF. Cupping reversal in pediatric glaucoma - evaluation of the retinal nerve fiber layer and visual field. Am J Ophthalmol. 2014;158(5):905-915.e1. doi:10.1016/j. ajo.2014.07.030 


\section{Publish your work in this journal}

Clinical Ophthalmology is an international, peer-reviewed journal covering all subspecialties within ophthalmology. Key topics include: Optometry; Visual science; Pharmacology and drug therapy in eye diseases; Basic Sciences; Primary and Secondary eye care; Patient Safety and Quality of Care Improvements. This journal is indexed on PubMed
Central and CAS, and is the official journal of The Society of Clinical Ophthalmology (SCO). The manuscript management system is completely online and includes a very quick and fair peer-review system, which is all easy to use. Visit http://www.dovepress.com testimonials.php to read real quotes from published authors. 